\title{
MYXOMYCETES EM CARNAUBEIRA (COPERNICIA PRUNIFERA (MILLER) H. E. MOORE, ARECACEAE)
}

\author{
Mitra Mobin ${ }^{1}$ \\ Laise de Holanda Cavalcanti ${ }^{2}$
}

Recebido em 16/09/1999. Aceito em 18/12/1999

\begin{abstract}
RESUMO - (Myxomycetes em carnaubeira (Copernicia prunifera (Miller) H. E. Moore, Arecaceae). Entre dezembro/1994 e setembro/1995 foram realizadas sete excursões ( 2 a 4 dias cada) ao Parque Nacional de Sete Cidades ( $4^{\circ} 5^{\prime}-4^{\circ} 15^{\prime}$ S, $41^{0} 30^{\prime}-41^{\circ} 40^{\prime} \mathrm{W}, 450 \mathrm{~m}$ alt.), em Piripiri, Piauí, visando coletar frutificações de Myxomycetes em indivíduos de Copernicia prunifera (Miller) H. E. Moore, estabelecidos em área de cerrado, na localidade Lagoa Seca. As amostras foram herborizadas e incorporadas ao herbário UFP (Universidade Federal de Pernambuco, Recife, PE), com duplicatas no herbário TEPB (Universidade Federal do Piauí, Teresina, PI). Registrou-se apreciável incidência (70\%) de Myxomycetes na carnaubeira, em estipe vivo e folhas mortas. Dezesseis espécies, pertencentes às Ceratiomyxaceae (Ceratiomyxa 1sp.), Didymiaceae (Diachea 1sp., Didymium 2spp.), Physaraceae (Craterium 1sp., Physarum 4spp.), Trichiaceae (Arcyria 2spp.) e Stemonitaceae (Comatricha 3spp., Macbrideola 1sp., Stemonitis 1sp.) foram registradas. Ceratiomyxaceae, representada por Ceratiomyxa fruticulosa (Mull.) Macbr., constitui primeira referência para o Estado do Piauí.
\end{abstract}

Palavras-chave - Myxomycetes, Arecaceae, Copernicia

\begin{abstract}
Myxomycetes on carnaubeira (Copernicia prunifera (Miller) H. E. Moore, Arecaceae). From December, 1994 to September, 1995, seven collecting trips (2-4 days each) were carried out in the "Parque Nacional de Sete Cidades" ( $4^{\circ} 5^{\prime}-4^{\circ} 15^{\prime} \mathrm{S}, 41^{\circ} 30^{\prime}-41^{\circ} 40^{\prime}$ 'W, $450 \mathrm{~m}$ alt.), Piripiri , Piauí State, Brazil, in order to collect Myxomycetes fruiting bodies on carnauba palm trees (Copernicia prunifera (Miller) H. E. Moore), established in a place called "Lagoa Seca" (savanna-like stunted vegetation). The samples were herborized and included in the UFP Herbarium (Universidade Federal de Pernambuco, Recife, PE) and the TEPB Herbarium (Universidade Federal do Piauí, Teresina, PI). In relation to the substrate, the "carnaubeira" showed a high incidence of Myxomycetes (70\%) on living stipitis and dead leaves. Sixteen species belonging to Ceratiomyxaceae (Ceratiomyxa 1sp.), Didymiaceae (Diachea 1sp., Didymium 2spp.), Physaraceae (Craterium 1sp., Physarum 4spp.), Trichiaceae (Arcyria 2spp.) and Stemonitaceae (Comatricha 3spp., Macbrideola 1sp., Stemonitis 1sp.), were registered. Ceratiomyxaceae, represented by Ceratiomyxa fruticulosa (Mull.) Macbr., is a new record for Piauí State.
\end{abstract}

Key words - Myxomycetes, Arecaceae, Copernicia

1 Departamento de Biologia SG1, CCN, Universidade Federal do Piauí, Campus Ministro Petrônio Portela, Ininga, CEP 6449-550, Teresina, PI, Brasil

2 Departamento de Botânica, CCB, Universidade Federal de Pernambuco, Av. Prof. Moraes Rego s/n, Cidade Universitária, CEP 50670-901, Recife, PE, Brasil, e-mail: 1hc@netpe.com.br 


\section{Introdução}

A carnaubeira (Copernicia prunifera (Miller) H. E. Moore) é uma palmeira economicamente importante, pois seu tronco serve para construções e das folhas, após secas, retira-se cera que é industrializada; as folhas são também utilizadas na confecção de cestas, chapéus, vassouras e outros artigos artesanais; as raízes, após queimadas e reduzidas a pó, podem substituir o sal de cozinha e são empregadas para fins medicinais (Lorenzi et al. 1996). Os frutos são comestíveis, tanto verdes, cozidos com leite, quanto maduros, consumidos crus e também servem como complemento da dieta de suínos, caprinos e bovinos. Possui, ainda, grande potencial paisagístico (Medeiros-Costa 1982).

Os indivíduos desta espécie possuem porte variável, os mais altos atingindo $15 \mathrm{~m}$; o tronco apresenta-se, em boa parte, caracteristicamente coberto pelas bases persistentes das folhas, dispostas helicoidalmente, tanto no sentido dextrógiro como no levógiro. As folhas são palmadas, arredondadas, de segmentos rijos, divididas até a metade da lâmina, com cobertura cerosa em ambas as faces; o pecíolo possui espinhos denteagudos. A inflorescência é interfoliar, variando de 1,5-3,0m compr., protegida por várias espatas tubulares sobrepostas. Os frutos da carnaubeira são ovóides, com aproximadamente $3,0 \mathrm{~cm}$ compr., verde-olivaarroxeados quando maduros (Medeiros-Costa 1982; Lorenzi et al. 1996).

No Brasil, a espécie distribui-se desde o Maranhão até os limites dos Estados da Bahia e Minas Gerais. As maiores populações concentram-se nos Estados do Piauí e Ceará, seguindo-se Rio Grande do Norte, Maranhão, Bahia, Paraíba e Pernambuco (Medeiros-Costa 1982).

No Parque Nacional de Sete Cidades, as carnaubeiras são encontradas na área denominada Lagoa Seca, com vegetação do tipo cerrado, onde alguns indivíduos apresentam vestígios da passagem do fogo por ocasião das queimadas que, em diferentes ocasiões, consumiram boa parte da vegetação.
A presença de Myxomycetes em vegetação de cerrado, no Brasil, tem sido relatada por Fidalgo et al. (1965), Gottsberger (1968), Cavalcanti $(1974$; 1977; 1985) e MaimoniRodella \& Gottsberger (1980), que não referem espécies de palmeiras como substrato nas áreas por eles estudadas. Por outro lado, Pôrto $e t$ al. (1985), Alves \& Cavalcanti (1996) e Mobin \& Cavalcanti (1999a) realizaram pesquisas sobre a ocorrência de Myxomycetes em palmeiras, encontradas em diferentes tipos de vegetação, registrando 41 espécies destes organismos sobre diferentes gêneros, como Acrocomia, Cocos, Copernicia, Elaeis, Mauritia e Orbygnia. No presente trabalho, efetuou-se o levantamento de espécies deste grupo de microrganismos ocorrentes em Copernicia prunifera desenvolvidos em área de cerrado, no Parque Nacional de Sete Cidades. Os resultados aqui relatados ampliam o número de espécies que utilizam palmeiras como substrato e contribuem para o conhecimento da distribuição dos Myxomycetes no Brasil, particularmente no Estado do Piauí.

\section{Material e métodos}

Dez indivíduos de carnaubeira foram localizados e estudados na área denominada Lagoa Seca, no Parque Nacional de Sete Cidades ( $4^{\circ} 5^{\prime}$ $4^{\circ} 15^{\prime} \mathrm{S}, 41^{\circ} 30^{\prime}-41^{\circ} 40^{\prime} \mathrm{W}, 450 \mathrm{~m}$ alt.) em sete viagens de coleta realizadas entre dezembro/1994 e setembro/1995, com dois a quatro dias de permanência na área de estudo em cada ocasião. Os indivíduos selecionados foram periodicamente observados, examinado-se o estipe desde a base até a altura de $1,65 \mathrm{~m}$; pesquisou-se a presença de Myxomycetes nos seguintes órgãos: estipe (vivo); folha (lâmina, pecíolo, bainha) e inflorescência (pedúnculo e espata) caídas ao chão.

Os esporocarpos encontrados foram coletados com seus respectivos substratos e, devidamente acondicionados (Cavalcanti 1974), transportados para o laboratório. As exsicatas foram depositadas no Herbário UFP, do Departamento de Botânica da Universidade Federal 
de Pernambuco (Recife, PE), com duplicatas no Herbário TEPB, do Departamento de Biologia da Universidade Federal do Piauí (Teresina, PI). A análise e identificação dos espécimes basearam-se nos trabalhos de Hagelstein (1944), Lister (1925), Martin \& Alexopoulos (1969), Robbrecht (1974) e Farr (1976), adotando-se o sistema de classificação de Martin et al. (1983) para gêneros e categorias supragenéricas.

\section{Resultados e discussão}

Nos 10 indivíduos de carnaubeira examinados durante o período de estudo, registrou-se $70 \%$ de incidência de frutificações de Myxomycetes, com o total de 34 amostras.

$\mathrm{O}$ maior número de assinalamentos foi efetuado na folha, quase sempre na lâmina, com poucos registros no pecíolo e bainha foliar. Não foram encontrados esporocarpos na espata e no pedúnculo da inflorescência. Raros registros foram obtidos no estipe, assinalando-se apenas Arcyria obvellata (Tab. 1).
Estudos realizados por Mobin \& Cavalcanti (1999a) no Parque Nacional de Sete Cidades, em buritizeiros (Mauritia vinifera $\mathrm{L}$. f.) ocorrentes em área de floresta, registraram 100\% de incidência de Myxomycetes, também predominando na folha, porém os esporocarpos foram mais freqüentes sobre o pecíolo. Nesta espécie de palmeira, entretanto, nenhum registro foi feito no estipe; comparando-se a arquitetura das duas espécies de palmeira, constata-se que o estipe da carnaubeira, por reter as bases das folhas, oferece melhores condições para o desenvolvimento dos Myxomycetes que o buritizeiro.

Foram assinaladas, no substrato estudado, 16 espécies, pertencentes às três subclasses de Myxomycetes, distribuídas em cinco famílias, predominando representantes dos gêneros Comatricha e Physarum (Tab.1). Dentre as famílias assinaladas, Ceratiomyxaceae, representada por Ceratiomyxa fruticulosa, é referida, pela primeira vez, para o Estado do Piauí.

Tabela 1. Ocorrência de Myxomycetes em Copernicia prunifera (Miller) H. E. Moore (Lagoa Seca, Parque Nacional de Sete Cidades, Piripiri, PI). (não registrado -; até dois registros +; entre 3-4 registros ++; entre 5-6 registros +++; primeiro relato para o Estado do Piaú *)

\begin{tabular}{|c|c|c|c|c|}
\hline \multirow[t]{2}{*}{ Táxons } & \multirow[t]{2}{*}{ Estipe } & \multicolumn{3}{|c|}{ Folha } \\
\hline & & Lâmina & Bainha & Pecíolo \\
\hline \multicolumn{5}{|l|}{ Ceratiomyxaceae * } \\
\hline Ceratiomyxa fruticulosa (Mull.) Macbr. * & - & + & - & - \\
\hline \multicolumn{5}{|l|}{ Trichiaceae } \\
\hline Arcyria denudata (L.) Wett. & - & - & + & - \\
\hline A. obvellata (Oeder.) Onsb. & + & - & - & + \\
\hline \multicolumn{5}{|l|}{ Stemonitaceae } \\
\hline Comatricha laxa Rost. & - & + & - & - \\
\hline C. cf. meandrispora Cast., Mor. \& Illa. & - & +++ & - & - \\
\hline C. pulchella (C. Bab.) Rost. & - & + & - & - \\
\hline Macbrideola martinii (Alexp. \& Beneke) Alexp. & - & + & - & - \\
\hline Stemonitis fusca Roth & - & + & - & - \\
\hline \multicolumn{5}{|l|}{ Physaraceae } \\
\hline $\begin{array}{l}\text { Craterium leucocephalum (Pers.) Ditmar var. scyphoides } \\
\text { (Cke \& Balf.) G. Lister }\end{array}$ & - & ++ & - & - \\
\hline Physarum flavicomum Berk. & - & + & - & - \\
\hline$P$ aff. galbeum Wing. & - & + & - & - \\
\hline P. aff. melleum (Berk. \& Br.) Mass. & - & + & - & - \\
\hline P. nucleatum Rex & - & ++ & - & - \\
\hline \multicolumn{5}{|l|}{ Didymiaceae } \\
\hline Diachea leucopodia (Bull.) Rost. & - & + & - & - \\
\hline Didymium clavus (Alb. \& Schw.) Rab. & - & + & - & - \\
\hline D. nigripes (Link) Fries & - & + & - & - \\
\hline
\end{tabular}


Alves \& Cavalcanti (1996) e Cavalcanti \& Putzke (1998) incluem $C$. fruticulosa e Physarum nucleatum na lista de espécies coletadas sobre palmeiras, no município do Crato, Ceará. Apesar de Alves \& Cavalcanti (1996) analisarem quatro espécies de palmeiras, dentre as quais Copernicia prunifera, a lista que apresentam não indica o substrato de ocorrência para cada espécie de Myxomycetes.

Farr (1960) cita a ocorrência de Physarum melleum e também de Arcyria denudata em palmeiras para Pernambuco, o primeiro coletado em Recife, sobre Cocos nucifera L. (coqueiro). Posteriormente, (Farr 1985) menciona a ocorrência de $C$. fruticulosa sobre palmeira não identificada, no Estado de Roraima, e de $A$. denudata para o Parque Nacional Eastern Dumoga-Bone, na Indonésia, novamente sem indicação da espécie de palmeira que serviu de substrato (Farr 1990). Pôrto et al. (1985) citam a presença de $A$. denudata sobre palmeiras dos gêneros Acrocomia, Cocos, Elaeis e Mauritia, para o Estado de Pernambuco; esta espécie também se inclui entre as registradas sobre Mauritia vinifera por Mobin \& Cavalcanti (1999a), no Parque Nacional de Sete Cidades. Lado \& Teyssiere (1998) incluem Elaeis guineensis Jacq. entre os substratos em que $A$. denudata foi encontrada, na Guiné Equatorial, assim como outras espécies de Trichiaceae.

Pôrto (1982), Pôrto et al. (1985) e Mobin \& Cavalcanti (1999a, 1999b) registraram a presença de Craterium leucocephalum e também de Physarum galbeum sobre palmeiras, ambos para Pernambuco e Piauí. Bononi et al. (1981) fazem referência a material desta última espécie, coletado no Parque Estadual das Fontes do Ipiranga, no município de São Paulo, SP, sobre folha de palmeira não identificada.

Physarum flavicomum é referido para o Brasil desde o início do século (Torrend 1915), incluindo a região nordeste, onde foi registrado sobre palmeiras por Pôrto et al. (1985), dentre as quais não se inclui a carnaubeira.

Diachea leucopodia é espécie cosmopolita, citada para diferentes regiões do Brasil.
Hochgesand \& Gottsberger (1996) comentam que esta espécie foi coletada em Botucatu (São Paulo) em área de floresta secundária, sobre folhas de palmeira não identificada. No Nordeste, este é o primeiro registro deste táxon sobre palmeiras. D. bulbillosa (Berk. \& Br.) A. Lister, recentemente referida para o Brasil (Mobin \& Cavalcanti 1999b), também foi registrada sobre palmeiras, em buritizeiros do Piauí (Mobin \& Cavalcanti 1999a).

Didymium nigripes, Comatricha laxa e Stemonitis fusca são referidas para o nordeste do Brasil sobre diferentes substratos; Pôrto et al. (1985) e Mobin \& Cavalcanti (1999c) citam sua ocorrência sobre palmeiras, porém não mencionam especificamente a carnaubeira.

Didymium clavus é freqüente em casca de árvores vivas no Estado de Pernambuco, e Mobin \& Cavalcanti (1999b) efetuaram o registro de sua presença em palmeiras no Piauí. Lado \& Teyssiére (1998) fizeram referência a esporocarpos dessa espécie encontrados sobre dendezeiros (E. guineensis) no Monte Alen, África Central, a $600 \mathrm{~m}$ de altitude.

Macbrideola martinii, Comatricha cf. meandrispora e Comatricha pulchella têm comumente como substrato madeira em decomposição e caules herbáceos, constituindo este o primeiro registro da ocorrência destas espécies sobre folhas de palmeira.

Merece destaque a ocorrência de Arcyria obvellata, esporulando em partes queimadas do estipe vivo (Tab. 1). Esta espécie é citada para a região nordeste, sob o binômio $A$. nutans (Bull.) Grev., coletada por Pôrto (1982) na mata de Dois Irmãos, Recife, PE, mas sua ocorrência sobre palmeiras é referida pela primeira vez no presente trabalho.

Dentre as espécies assinaladas na carnaubeira, 11 têm registro de ocorrência sobre diferentes gêneros de palmeiras, porém a maioria dos autores não indica a espécie que lhes serviu de substrato. Considerando que no Brasil apenas Alves \& Cavalcanti (1996) citam a carnaubeira como substrato para Myxomycetes, dentre as espécies aqui relatadas, 14 estão sen- 
do assinaladas pela primeira vez sobre esta espécie de palmeira, elevando para 56 o número de representantes da classe encontrados em indivíduos da família Arecaceae no Brasil.

\section{Agradecimentos}

As autoras agradecem o apoio logístico proporcionado pela direção do Parque Nacional de Sete Cidades e pelo Departamento de Biologia da Universidade Federal do Piauí, bem como bolsas de Mestrado, Desenvolvimento Regional e Produtividade em Pesquisa concedidas pela CAPES e CNPq às autoras, e auxílio financeiro proporcionado pela FACEPE.

\section{Referências bibliográficas}

Alves, M. H. \& Cavalcanti, L. H. 1996. Myxomycetes em palmeiras (Arecaceae). Acta Botanica Brasilica 10(1): 1-7.

Bononi, V. L. R.; Trufem, S. F. B. \& Grandi, R. A. P. 1981. Fungos macroscópicos do Parque Estadual das Fontes do Ipiranga, São Paulo, Brasil, depositados no Herbário do Instituto de Botânica. Rickia 9: 37-53.

Cavalcanti, L. H. 1974. Mixomicetos corticícolas do cerrado de Emas (Pirassununga-São Paulo). Dissertação de Mestrado. Universidade de São Paulo, São Paulo.

Cavalcanti, L. H. 1977. Mixomicetos do cerrado II - Análise comparativa das espécies encontradas no cerrado protegido e no queimado anualmente. Pp. 129-140. In: Anais do XXVIII Congresso Nacional de Botânica. EPAMIG, Belo Horizonte.

Cavalcanti, L. H. 1985. Mixomicetos do cerrado III - Revisão de literatura. Pp. 173-179. In: Anais do XXXIII Congresso Nacional de Botânica. EMBRAPA, Brasília.

Cavalcanti, L. H. \& Putzke, J. 1998. Myxomycetes da chapada do Araripe (Crato-CE, Brasil). Acta Botanica Brasilica 12(3): 253-261.

Farr, M. L. 1960. The Myxomycetes of the IMUR Herbarium with special reference to Brazilian species. Publicações do Instituto de Micologia 184: 1-54.

Farr, M. L. 1976. Myxomycetes. Flora Neotropica. Monograph 16. New York Botanical Garden, New York.

Farr, M. L. 1985. Notes on Myxomycetes IV. Species collected in Brazil and Japan. Nova Hedwigia 41: 167-175.

Farr, M. L. 1990. Myxomycetes. Pp. 169-171. In: G. J. Samuels. Contributions toward a mycobiota of Indonesia: Hypocreales, Synnematous Hyphomycetes, Aphyllophorales, Phragmobasiodiomycetes and Myxomycetes. New York Botanical Garden, New York.
Fidalgo, O.; Fidalgo, M. E. P. K. \& Furtado, J. S. 1965. Fungi of the "cerrado" region of São Paulo. Rickia 2: 55-65.

Gottsberger, G. 1968. Myxomyceten aus Bahia und Goiás. Nova Hedwigia 15: 361-368.

Hagelstein, R. 1944. The Mycetozoa of North America. Published by the Author, Mineola.

Hochgesand, E. \& Gottsberger, G. 1996. Myxomycetes from the State of São Paulo, Brazil. Boletim do Instituto de Botânica 10: 1-46.

Lado, C. \& Teyssiere, M. 1998. Myxomycetes from Equatorial Guinea. Nova Hedwigia 67(3/4): 421-441.

Lister, A. 1925. A monograph of the Mycetozoa. British Museum Natural History, London.

Lorenzi, H.; Souza, H. M.; Medeiros-Costa, J. T. \& Cerqueira, N. V. B. 1996. Palmeiras do Brasil nativas e exóticas. Plantarum, São Paulo.

Maimoni-Rodella, R. C. S. \& Gottsberger, G. 1980. Myxomycetes from the forest and the cerrado vegetation in Botucatu, Brazil: a comparative ecological study. Nova Hedwigia 34: 207-247.

Martin, G. W. \& Alexopoulos, C. J. 1969. The Myxomycetes. University of Iowa Press, Iowa.

Martin, G. W.; Alexopoulos, C. J. \& Farr, M. L. 1983. The genera of Myxomycetes. University of Iowa Press, Iowa.

Medeiros-Costa, J. T. 1982. As palmeiras (Palmae) nativas em Pernambuco, Brasil. Dissertação de Mestrado. Universidade Federal Rural de Pernambuco, Recife.

Mobin, M. \& Cavalcanti, L. H. 1999a. Myxomycetes sobre buriti (Mauritia vinifera L. f., Arecaceae). Revista da Universidade do Amazonas, Série Ciências Biológicas 2/3(1-2): 43-51.

Mobin, M. \& Cavalcanti, L. H. 1999b. Physarales (Myxomycetes) do Parque Nacional de Sete Cidades, Piauí, Brasil. Hoehnea 26(1): 1-14 .

Mobin, M. \& Cavalcanti, L. H. 1999c. Stemonitales (Myxomycetes) do Parque Nacional de Sete Cidades (Piauí, Brasil). Acta Botanica Brasilica 13(2):139. 148.

Pôrto, K. C. 1982. Myxomycetes da Mata de Dois Irmãos, Recife-Pernambuco. Dissertação de Mestrado. Universidade Federal Rural de Pernambuco, Recife.

Pôrto, K. C.; Cavalcanti, L. H. \& Correia, A. M. S. 1985. Incidência de Myxomycetes em Palmae. Pp. 181-187. In: Anais do XXXIII Congresso Nacional de Botânica. EMBRAPA, Brasília.

Robbrecht, E. 1974. The genus Arcyria Wiggers (Myxomycetes) in Belgium. Bulletin du Jardin Botanique National de Belgique 44: 303-353.

Torrend, C. 1915. Les Myxomycétes du Brésil, connus jusqu'ici. Broteria, Série Botânica 13: 72-88. 